\title{
Exploring the Impact of Travel Vlogs on Prospect Tourists: A SOR Based Theoretical Framework
}

\author{
Wenliang $\mathrm{Li}^{\left({ }^{\square}\right)}$ (D), Yoo Ri Kim (D), Caroline Scarles (D), \\ and Anyu Liu $\mathbb{D}$ \\ School of Hospitality and Tourism Management, University of Surrey, \\ Guildford, Surrey, UK \\ \{wenliang. li, yoori.kim, \\ c.scarles, anyu.liu\}@surrey.ac.uk
}

\begin{abstract}
In recent years, travel vlogs are prevalent on social media, they are projected as an important marketing tool to attract tourists to destinations in the post-COVID-19 era. However, the underlying mechanism of how travel vlogs affect prospective tourists' behaviours remains unclear. To address this gap, this paper discusses the applicability of the Stimulus-Organism-Response (SOR) model to travel vlog research and proposes a SOR based theoretical framework. Moreover, this paper highlights the increasing trend of the SOR model in both etourism and wider tourism and hospitality research.
\end{abstract}

Keywords: Travel vlog $\cdot$ Stimulus-Organism-Response $\cdot$ Theoretical framework

\section{The Travel Vlog Phenomenon}

In recent years, vlog (video blog), a new type of user-generated content (UGC) which refers to a casual, conversational short video format featuring a person talking directly and intimately to the camera and relating their feelings and life experiences in a raw and unfiltered way has proliferated on social media [19]. Travel is becoming the most popular vlog topic [14], and travel vlogs have also become the most popular type of travel videos viewed on YouTube, receiving four times more social engagement than other types of travel videos and almost half of the travel subscriptions [2]. Given the wealth of online traffic, travel vlogs have huge marketing potential for destinations to attract tourists [2]. However, they are overlooked in the current literature [2].

After COVID-19, social media marketing is expected to play an important role in destination marketing, and travel vlogs are projected to be a great strategy to heighten the audiences' travel desires [13]. Therefore, it is necessary to understand how travel vlogs influence prospective tourists' behaviours so that it can help destinations better attract tourists. However, most studies have emphasised the direct link between travel vlogs and the audiences' subsequent behaviours but overlooked the potential underlying 
mechanism, which remains a critical research gap [2]. Hence, this paper proposes to use the SOR model as a useful theoretical base to build up a framework to further explore the impact of travel vlogs on prospective tourists' behaviours.

\section{The SOR Model and Its Applicability to Travel Vlog Research}

\subsection{Overview of the SOR Model}

The SOR model was firstly proposed by Mehrabian and Russell [8], it was rooted in environmental psychology and developed from the classic Stimulus-Response model in behaviourism [10]. The SOR model suggests that a stimulus induces an individuals' internal states, which in turn leads to certain behavioural responses [20]. In the SOR model, stimulus is conceptualised as an influence that arouses the individual. Organism is defined as "the psychological process that occurs in the individual's mind when interacting with the stimulus" [20, p. 467], playing an intervening or mediating role in the model. Originally, organism refers to an individual's emotional states while nowadays it is commonly represented by both emotional and cognitive states [5]. Response could be explained as the final behavioural outcome of an individual.

The SOR model has been mostly applied to retail research while it is increasingly being applied to tourism and hospitality research especially to the e-tourism context to predict consumer behaviour [5]. It has been observed that existing SOR based tourism and hospitality studies have attempted to examine how attributes of various information and communication technologies (ICTs) (e.g., booking websites, mobile apps, and virtual realities) impact on tourists' behavioural responses. Recently, some SOR based tourism and hospitality studies have explored the impact of travel UGC as an external stimulus [3], such as Facebook pages and Instagram posts. However, travel UGC videos like vlogs have not yet been studied in articles we reviewed, which remains a gap. Compared to textual and static pictural information, individuals' responses to videos could be different. Thus, a tailored SOR model which fits the features of videos is underdeveloped in tourism and hospitality research.

\subsection{Applicability of the SOR Model to Travel Vlog Research}

The rise of the application of the SOR model in recent e-tourism and wider tourism and hospitality studies inspires this paper to explore the applicability of the SOR model to travel vlog research. The SOR model is suitable for travel vlog research for two reasons.

Firstly, as a systematic approach to conceptualising linkages between key constructs underpinning emotions, the SOR model has been most extensively used when examining consumer behaviour from the emotional perspective. The theory of reasoned action, technology acceptance model, and theory of planned behaviour are the most common consumer behaviour models [9]. However, they are critiqued for being too limited when it comes to explaining the emotional side of consumer behaviour because their main assumption is that individuals are rational in their decision-making processes 
and actions [9]. By contrast, the SOR model can be used as a supplement to the traditional lens of rational factors to explain consumer behaviour. Recent empirical evidence demonstrated that travel vlog audiences are more likely to be affected by emotional states rather than rational facts [2]. Therefore, as a more emotional framework, the SOR model may predict travel vlog audiences' behaviour well.

Secondly, as a systemised theoretical framework with a parsimonious and structured manner, the SOR model has strong wide applicability. Sultan et al. [10] stressed that the SOR model is flexible to allow for various factors like internal and external stimuli, experiential and non-experiential organisms (e.g., emotion, perception/feeling) and responses (e.g., intention, behaviour). Due to its powerful flexibility, the SOR model provides researchers with a good theoretical base to tailor the general consumer behaviour model, reflecting its intuitive and powerful exploratory nature. Compared to the wider tourism and hospitality research and the application of the SOR model, research on travel vlogs around is underexplored [2]; the SOR model is therefore suitable for being applied to exploratory travel vlog studies given its strengths in eliciting emotions of tourists and their behavioural responses.

\section{Development of a SOR Based Theoretical Framework}

A SOR based theoretical framework to explore how travel vlogs could influence prospective tourists' behaviours is presented as follows. As depicted in Fig. 1, this framework proposes travel vlog's storytelling as the stimulus, perceived intimacy, perceived authenticity, presence, and emotions as the organism, destination image and destination visit intention as the response, and personal traits as the moderator.

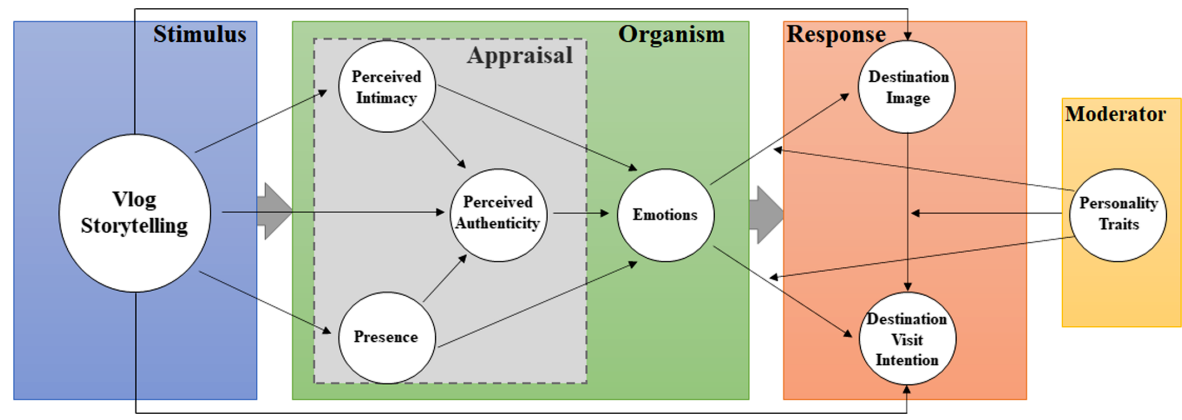

Fig. 1. A SOR based theoretical framework

Zhang et al. [18] revealed that a compelling story provides the stimuli that evoke the audiences' sensations, cognitive, and behavioural responses. As vlog is particularly called as "storytelling video" [4], and storytelling is believed as the key to a vlog's success in attracting the audiences' attention [16]. It is worthy to consider it as a key influence of a vlog on the audiences. In the organism construct of this framework, emotions elicited by vlogs are designed to be the core organismic factor. According to 
Tuerlan et al. [11], appraisal theories of emotions, which argue that an individual's emotional responses are the outcome of a subjective mental evaluation of the stimulus rather than the direct result of the stimulus is being an agenda for future emotion research in the tourism and hospitality. Given that, this framework proposes perceived intimacy, perceived authenticity, and presence ("3Ps") as the direct antecedents of emotions, representing the stimulus appraisals by an individual. Literature shows that "3Ps" have been used as appraisals of the stimulus and vlogs can facilitate the audiences" "3Ps" [7, 12, 17].

Prospective tourists' perceived destination image and destination visit intention together consist of the response construct of this framework. They both are crucial for the success of destination marketing. As the result of a perceptual and cognitive process, destination image is formed from information sources [6]; it is an important predictor of destination visit intention [1]. Destination visit intention is an intentional behaviour commonly used to predict prospective tourists' actual visit. In terms of the inclusion of a moderator in this framework, it may help to find out the reasons behind the resistance to use travel vlogs to make destination visits. In the context of social media use, personality traits have been widely used as moderating variables to predict users' behaviours [15].

\section{Conclusion}

As travel vlog research is just emerging, the underlying mechanism of how travel vlogs affect prospective tourists' behaviours is still unclear. This paper captures the increasing trend of the application of the SOR model in e-tourism and wider tourism and hospitality studies and discusses its applicability to travel vlog research. The SOR model was found that is good at exploring prospective tourists' behavioural responses associated with travel vlog watching from the emotional perspective and is flexible for testing various variables. Therefore, based on the SOR model, this paper proposes a theoretical framework to explore how travel vlogs' storytelling triggers prospective tourists' destination visit intention. The originality of the research is in two-folds. First, this is the first attempt to introduce digital storytelling as the stimulus in the SOR model to capture the nature of vlog as a new video medium. Second, the other novelty of the proposed framework is the integration of appraisal theories of emotions with the SOR model which could better capture the emotional feedback of vlog viewers.

This conceptual framework will be further examined in future research, the validation of this framework is expected to extend the SOR based tourism and hospitality literature, help destination marketers to better communicate with prospective tourists and trigger them to make visits, and provide a guideline for travel vloggers to improve their storytelling so that they can better engage with their audiences. Besides that, this research may foster the collaboration between destination marketers and vloggers to create favourable travel vlogs for destination promotion. Lastly, it should be noted that a large number of recent SOR based tourism and hospitality articles have been observed, which demonstrates that the capability of the SOR model in predicting tourist behaviour has been gaining more and more recognition and the SOR model seems to be becoming a mainstream theory employed in tourism and hospitality research. 


\section{References}

1. Afshardoost M, Eshaghi MS (2020) Destination image and tourist behavioural intentions: a meta-analysis. Tour Manage 81:104154

2. Cheng Y, Wei W, Zhang L (2020) Seeing destinations through vlogs: implications for leveraging customer engagement behavior to increase travel intention. IJCHM 32(10):32273248

3. Cheung ML, Leung WK, Cheah J-H, Ting H (2021) Exploring the effectiveness of emotional and rational user-generated contents in digital tourism platforms. J Vacat Mark 1-19

4. Huan TJ, Chin TA, Sulaiman Z, Zakuan N (2020) Review the relationships between antecedents of parasocial interaction and branding in video blog. In: ICoEC2020 eProceedings, pp 65-69

5. Kim MJ, Lee C-K, Jung T (2020) Exploring consumer behavior in virtual reality tourism using an extended Stimulus-Organism-Response model. J Travel Res 59(1):69-89

6. Lopes SDF (2011) Destination image: origins, developments and implications. Pasos 9 (2):305-315

7. Luoma-aho V, Pirttimäki T, Maity D, Munnukka J, Reinikainen H (2019) Primed authenticity: how priming impacts authenticity perception of social media influencers. Int $\mathrm{J}$ Strateg Commun 13(4):352-365

8. Mehrabian A, Russell JA (1974) An approach to environmental psychology. The MIT Press, Cambridge

9. Moon MA, Khalid MJ, Awan HM, Attiq S, Rasool H, Kiran M (2017) Consumer's perceptions of website's utilitarian and hedonic attributes and online purchase intentions: a cognitive-affective attitude approach. Span J Mark - ESIC 21(2):73-88

10. Sultan P, Wong HY, Azam MS (2021) How perceived communication source and food value stimulate purchase intention of organic food: an examination of the Stimulusorganism-Response (SOR) model. J Clean Prod 312:127807

11. Tuerlan T, Li S, Scott N (2021) Customer emotion research in hospitality and tourism: conceptualization, measurements, antecedents and consequences. Int $\mathbf{J}$ Contemp Hosp Manage 33(8):2741-2772

12. Wang X, Chang B (2020) The impact of the audience's continuance intention towards the vlog: focusing on intimacy, media synchronicity and authenticity. Int J Contents 16(2):6577

13. Xie X (2021) Research on social media tourism marketing in the post-epidemic period. Jurnal Audiens 2(2):273-280

14. Xinhua (2019) Survey shows more than half of Chinese watch vlogs. China Daily. http:// www.chinadaily.com.cn/a/201906/08/WS5cfb5ee8a31017657723010d.html

15. Yu T-K, Lee N-H, Chao C-M (2020) The moderating effects of young adults' personality traits on social media immersion. Front Psychol 10:554106

16. Zhang $\mathrm{H}$ (2020) Analysis on vlog story-telling techniques and advertisement. In: Proceedings of ICHESS 2020. ASSEHR, vol 496. Alantis Press, pp 68-72

17. Zhang H (2018) Evoking presence in vlogging: a case study of U.K. beauty blogger Zoe Sugg. FM 23(1)

18. Zhang W, Kim J, Kim H, Fesenmaier DR (2019) The tourism story project developing the behavioral foundations for an AI supporting destination story design. e-Rev Tour Res 17 (2):170-187 
19. Zhou J (2018) Short and sweet, the new craze for the video generation. China Daily. http:// www.chinadaily.com.cn/cndy/2018-06/16/content_36401015.htm

20. Zhu L, Li H, Wang F-K, He W, Tian Z (2020) How online reviews affect purchase intention: a new model based on the Stimulus-Organism-Response (S-O-R) framework. Aslib J Inf Manage 72(4):463-488

Open Access This chapter is licensed under the terms of the Creative Commons Attribution 4.0 International License (http://creativecommons.org/licenses/by/4.0/), which permits use, sharing, adaptation, distribution and reproduction in any medium or format, as long as you give appropriate credit to the original author(s) and the source, provide a link to the Creative Commons license and indicate if changes were made.

The images or other third party material in this chapter are included in the chapter's Creative Commons license, unless indicated otherwise in a credit line to the material. If material is not included in the chapter's Creative Commons license and your intended use is not permitted by statutory regulation or exceeds the permitted use, you will need to obtain permission directly from the copyright holder.

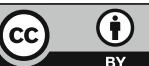

